\title{
Estudios controlados en humanos de éxtasis
}

\author{
Magí Farré Albadalejo ${ }^{1,2}$, Pere N. Roset Arissóo, ${ }^{1,2}$, Rafael de la Torre Fornelli, \\ Sandra Poudevida Jürschik', Sergio Abanades León ${ }^{1,2}$; Ana Ma Peiró Peiró ${ }^{1,2}$, \\ Yolanda Álvarez García ${ }^{1,2}$, Nieves Pizarro lozano ${ }^{1,3}$, Mireia Segura Agullóo ${ }^{1,3}$, \\ Jordi Camí MorelL ${ }^{1,3}$
}

'Unitat de Farmacología. Institut Municipal d'Investigació Mèdica (IMIM), Barcelona

${ }^{2}$ Universitat Autònoma de Barcelona ${ }^{3}$ Universitat Pompeu Fabra, Barcelona

Enviar correspondencia: Magí Farré, Unitat Farmacologia, IMIM. Doctor Aiguader, 80. 08003 Barcelona. Tel 93 2211009; Fax 93 2213237; e-mail: mfarre@imim.es

\section{Resumen}

Objetivo: recopilar y analizar la información sobre los efectos de la MDMA obtenida en estudios controlados en humanos. Método: la mayoría de ensayos analizados han sido de diseño enmascarado a doble ciego, cruzados, con asignación aleatoria a la secuencia de tratamientos, y controlados con placebo y/o fármaco patrón. Resultados: además del incremento de la presión y frecuencia cardíaca, la MDMA incrementa el consumo miocárdico de oxígeno sin efectos inotrópicos y puede incrementar el intervalo QTc en el ECG. El aumento de temperatura no fue significativo frente a placebo. Produce euforia, bienestar y activación. No se han descrito ni alucinaciones ni ilusiones. En el EEG aumenta la actividad beta y disminuye la alfa. Aumenta de forma dosis-dependiente las concentraciones de ACTH, cortisol, prolactina, dihidroepiandrosterona y vasopresina. Inmunológicamente su efecto parece ser inmunosupresor. Su farmacocinética parece ser no-lineal con tendencia a la acumulación a dosis alta. Conclusiones: los estudios controlados han permitido conocer mejor la MDMA, profundizando en sus efectos subjetivos, y aportando datos originales en cuanto a cambios hormonales e inmunológicos. Se necesitan más estudios para determinar científicamente los efectos y toxicidad de la MDMA.

Palabras claves: MDMA, farmacocinética, efectos farmacológicos, ensayo clínico controlado.

\section{Summary}

Objective: to compile and analyse knowledge about the pharmacodynamics and pharmacokinetics of MDMA from controlled clinical trials in humans. Methods: the typical design of the trials was masked (double blind), randomized, cross-over, and controlled with placebo and/or an active drug. Results: MDMA increases the heart rate, arterial blood pressure, oxygen consumption by the heart and produced an enlargement of the QTC interval in the ECG. No statistical differences were found with placebo in the increase of body temperature. MDMA induce euphoria, wellbeing and activation. Neither ilusions nor hallucinations were described. An increase of beta activity and a decrease of alpha activity in the EEG was seen. A dose-dependent increase in the plasma levels of $\mathrm{ACTH}$, cortisol, prolactin, dehydroepiandrosterone and vasopresine was observed. Immunogically, MDMA posses an immunosupresor effect. Its pharmacokinetic properties seems to be non-lineal, with a tendency to accumulation at high doses. Conclusion: the results obtained in these controlled clinical trials increase our previous knowledge on MDMA and add new data related to its hormonal and immunological effects. Further studies are needed in order to better understand the effects and toxicity of MDMA.

Key Words: MDMA, pharmacokinetics, pharmacological effects, controlled clinical trial 


\section{LA INVESTIGACIÓN DE LA FARMACOLO- GÍA HUMANA DE LAS DROGAS}

$\mathbf{L}$ a investigación de la farmacología clínica de las drogas comprende el estudio de su farmacodinamia (mecanismo de acción, efectos farmacológicos, adversos y terapéuticos) y de su farmacocinética (absorción, distribución, metabolismo y excreción) en humanos. El conocimiento de los efectos y la farmacocinética de las drogas de abuso es distinto, según sean medicamentos o no lo sean. En el primer caso existirán investigaciones experimentales previas en humanos, mientras que serán escasas e incluso inexistentes en el segundo.

Cuando las drogas de abuso son (o fueron) medicamentos, es decir que se utilizan para el tratamiento o prevención de enfermedades, ya han sido investigadas como tales y han sido objeto de un desarrollo farmacéutico estándar. Este incluye estudios en animales y humanos que permitan su registro y la aprobación por las autoridades sanitarias. En el caso de los fármacos con acción sobre el sistema nervioso central, si son derivados de sustancias que pueden tener potencial de abuso o dependencia, o si pueden utilizarse para el tratamiento de estos dos trastornos, es obligatorio realizar estudios en animales para evaluar el potencial de abuso y dependencia y si es el caso reproducirlos en humanos. Estos estudios permitirán decidir si deben existir limitaciones para su prescripción, dispensación y uso. La sustancia podrá ser incluida en su caso en algunas de las listas de sustancias estupefacientes o psicotropas. Algunos de los fármacos objeto de esta monografía pertenecen al grupo de medicamentos, comercializados en uno o varios países, con una indicación terapéutica concreta. Por ejemplo la ketamina, el oxibato sódico (gammahidroxibutirato sódico o GHB) o el dextrometorfano han sido estudiados en humanos como medicamentos y se conocen muchos aspectos de su farmacodinamia, farmacocinética, utilidad terapéutica y toxicidad. También es cierto que los aspectos relacionados con su potencial de abuso, y los efectos subjetivos que producen a las dosis habitualmente utilizadas por los consumidores, no fueron investigados a fondo ya que se trata de fármacos antiguos y no se sospechó que podrían llegar a ser objeto de abuso (Holland, 2001).

Cuando una droga no es un medicamento, la mayoría de datos provienen de estudios descriptivos o series de casos en que se observan las complicaciones agudas o crónicas tras su uso, y/o se describen los efectos típicos de la sustancia mediante entrevistas o encuestas a los consumidores. Esta es la forma más común para conocer los síntomas y las enfermedades provocadas por la sustancia. El saber es parcial, ya que al tratarse de sustancias ilegales es casi imposible conocer la dosis ingerida. Además, muchas veces las intoxicaciones agudas son por múltiples sustancias y sólo se conocen los efectos en una parte de la población, la que acude a solicitar asistencia sanitaria por presentar complicaciones médicas. En el caso de la farmacocinética los datos son anecdóticos e incompletos, con un valor limitado a la toxicología clínica o aspectos forenses. En cuanto a las descripciones o encuestas a los consumidores, valen las limitaciones antes expuestas en cuanto a dosis, pureza, combinaciones y ambiente de consumo.

Estas descripciones se basan en casos únicos o series pequeñas. Aunque en ocasiones estos relatos se basan en un diseño experimental antes-después, nunca son estudios controlados y con garantías de garantía científica.

Ambas aproximaciones tienen limitaciones evidentes. Muchos de los efectos de las drogas no pueden determinarse en las intoxicaciones, ni en las encuestas o entrevistas, y mucho menos su farmacocinética. Al tratarse de sustancias que no son medicamentos, el conocimiento científico sobre sus acciones y efectos en humanos es muy limitado, dándose la paradoja que, por ejemplo, mientras que el consumo de la mayoría de las drogas de síntesis es muy elevado, el número de investigaciones farmacológicas en humanos es casi inexistente. 
Para conocer de forma adecuada la farmacología humana de una droga se precisan estudios experimentales, en los que se administren estas sustancias en un contexto conocido, a ser posible en condiciones de ensayo clínico aleatorio, enmascarado y controlado con placebo y/o sustancia patrón. En estos estudios puede determinarse de forma precisa la causalidad de los efectos observados o narrados por los sujetos. Además permiten el estudio de la farmacocinética del fármaco en sangre, orina y otras matrices biológicas (sudor, saliva, cabello).

La propia naturaleza experimental de estos estudios hace que deban realizarse siguiendo las recomendaciones de la Declaración de Helsinki, respetando los principios bioéticos básicos de no maleficencia, justicia, beneficencia y autonomía. El objetivo es proteger a los individuos de posibles daños físicos o psicológicos. Por este motivo los estudios de farmacología humana de las drogas se realizan a dosis única o tras pocas dosis, y se seleccionan como participantes a sujetos con experiencia previa con drogas que consienten voluntariamente a participar. Cuentan con la aprobación de un Comité Ético de Investigación Clínica (CEIC) y de las autoridades sanitarias (Agencia Española del Medicamento) en el caso España. Los estudios experimentales de farmacología humana ayudan a entender los efectos de las drogas pero deben sumarse a los estudios observacionales antes mencionados. Los estudios observacionales servirán para clarificar aquellos aspectos que son imposibles de abordar con el diseño anterior y permitirán una aproximación realista a las condiciones habituales de consumo. Son imprescindibles para conocer las pautas de consumo, las razones del uso y la importancia de las situaciones, las asociaciones con otras drogas, los efectos subagudos o crónicos, y las complicaciones médicas a corto, medio y largo plazo. Además actuarán como señal de alarma para detectar problemas que después pueden ser abordados en experimentos planificados. Debe recordarse que de acuerdo con las proposiciones de la Medicina Basada en Pruebas ( o Evidencias), los diferentes tipos de estudios proporcionan un nivel de calidad de causalidad distinto y que debe conocerse para poder interpretar de forma adecuada los resultados (Tabla 1). Esta clasificación es aplicable sobre todo para evaluar los efectos farmacológicos y terapéuticos y la farmacocinética, pero resulta menos adecuada para ser aplicada en los efectos indeseables de baja incidencia. Debe insistirse que un tipo de estudio no excluye al otro y que ambos deben coexistir, ya que los estudios experimentales y los observacionales son complementarios (Baños y Farré, 2002).

\section{LA INVESTIGACIÓN CON MDMA}

En general, excepto en los Estados Unidos de Norteamérica, la investigación en huma-

Tabla 1. Gradación de mayor a menor de la calidad del nivel de la evidencia en función del rigor científico del estudio (Agència d'Avaluació de Tecnologia Mèdica de Catalunya).

1. Metaanálisis de ensayos controlados y aleatorizados

2. Ensayo controlado y aleatorizado de muestra grande

3. Ensayo controlado y aleatorizado de muestra pequeña

4. Ensayo prospectivo controlado no aleatorizado (controles coincidentes en el tiempo)

5. Ensayo prospectivo controlado no aleatorizado (controles históricos)

6. Estudios de cohorte

7. Estudios de casos y controles

8. Series clínicas no controladas. Estudios descriptivos. Comités de expertos

9. Anécdotas o casos únicos 
nos de las drogas de abuso encuentra dificultades difíciles de superar. Los propios estados y las Naciones Unidas (ONU) son muy recelosos con el uso de drogas y por ello limitan su investigación en humanos. Para algunas sustancias se ha dado la circunstancia de que han sido prohibidas sin contar con fundamento ni conocimiento científico de sus efectos en humanos. Entre ellas se encuentra la 3,4-metilenodioximetanfetamina (MDMA o comúnmente éxtasis). Se sintetizó en 1912 como producto intermedio, y parece que no fue investigada hasta principios de los años cincuenta, en que se evaluó su toxicidad en animales. En los años setenta la re-sintetizaron químicos como Alexander Shulgin que autoexperimentaron con ella. Además de los efectos típicos de los psicoestimulantes (euforia, bienestar, activación), les sorprendieron sus propiedades empatizantes. En ese momento la MDMA no era ilegal. La fama de la MDMA fue creciendo, algunos psiquiatras empezaron a utilizarla en sesiones de psicoterapia y se difundió su empleo recreativo como droga de abuso. En 1985, a la vista de que el consumo era importante en algunas ciudades, el gobierno norteamericano propuso prohibir la MDMA y lo consiguió (Holland, 2001). Hasta ese momento no existía ningún estudio sistemático ni riguroso de sus efectos, todo se basaba en algún estudio experimental no controlado y descripciones de los consumidores.

Algo distinta es la historia de la 3,4-metilenodioxianfetamina (MDA tenanfetamina 0 comúnmente píldora del amor), que fue patentada y estudiada en humanos en 1958 como anorexígena, pero la investigación fue abandonada por sus propiedades psicoactivas. La MDA fue utilizada en los sesenta por algunos psiquiatras durante sesiones de psicoterapia y se inició su consumo recreativo. A principios de los setenta fue declarada ilegal en los EEUU (Holland, 2001).

Desde inicios de los noventa el consumo de MDMA ha sido fuente de preocupación en Europa, Norteamérica y Australia. Su consumo se asocia a ambientes festivos (raves) y determinados géneros musicales. En Espa- ña se inició con la llamada ruta del bakalao y la música techno (Camí y Farré, 1996). Se conoce que probablemente más de un centenar de miles de jóvenes toman una o varias pastillas de éxtasis cada fin de semana en nuestro país. En el Reino Unido esta cifra supera el medio millón. Resulta paradójico que mientras millones de jóvenes tomaban de forma incontrolada MDMA, las autoridades sanitarias no permitían la investigación en situaciones controladas. No se conocía su metabolismo o su farmacocinética en humanos, no estaba claro si tenía propiedades alucinógenas, y no se sabía que podía ocurrir si se administraba conjuntamente con otras sustancias. La ignorancia de aspectos básicos de una de las drogas más consumidas llevó a que a mitad de los noventa diferentes administraciones permitieran iniciar estudios experimentales de farmacología clínica de la MDMA en humanos. La autorización se concedió primero en Estados Unidos (grupos del Dr. Grob, Dr. Jones y Dr. Schuster) y Suiza (Dr. Vollenweider) y después en España (Dr. Camí), Reino Unido (Dr. Henry) y Holanda (Dr. Riedel). También se han realizado estudios experimentales con 3,4-metilenodioxeetilanfetamina (MDE o Eva) en Alemania (Dra. Gouzoulis-Mayfrank) (Gouzoulis-Mayfrank et al, 1999a; Gouzoulis-Mayfrank et al, 1999b). Además se han autorizaron ensayos clínicos para estudiar los posibles efectos terapéuticos de la MDMA en España (Drs. Sopelana y Bouso) y están pendientes de aprobación en los EEUU e Israel (en el momento de cerrar este capítulo)(Kleinman y Holland, 2001).

\section{ESTUDIOS CONTROLADOS DE ADMINIS- TRACIÓN DE MDMA EN HUMANOS}

Para poder realizar una evaluación científica de una sustancia se requiere que puedan neutralizarse las expectativas e influencias de los sujetos e investigadores, para ello se precisa de la administración enmascarada de los tratamientos (doble ciego) y la asignación aleatoria. La inclusión de placebo y/o un fármaco 
de referencia permitirá evaluar los efectos de forma comparativa y conocer aquellos que son propios de la sustancia activa. Todo ello justifica la realización de estudios controlados bajo las condiciones antes expuestas cuando se quieren evaluar los efectos farmacológicos de una sustancia (Baños y Farré, 2002).

En el caso de la farmacocinética debe administrase el fármaco en condiciones estandarizadas, recogiendo muestras de fluidos biológicos a tiempos adecuados para poder definir los principales parámetros de interés que pueden relacionarse con los efectos o la toxicidad. En estos estudios se determinan de forma exacta las concentraciones del fármaco y/o sus metabolitos en los diferentes fluidos biológicos, por este motivo las exigencias metodológicas son menores al considerarse los resultados poco influenciables por los sujetos y/o investigadores. Los diseños experimentales simples pueden ser adecuados. Aún así, la mayoría de estudios se realizan enmascarados al incluir simultáneamente evaluaciones de efectos farmacológicos.

Los grupos antes citados han realizado diferentes ensayos clínicos en los que se ha administrado MDMA en grupos reducidos de voluntarios sanos. Los protocolos fueron aprobados por un Comité Ético (y las autoridades sanitarias según la legislación de cada país), los sujetos fueron informados de los riesgos y firmaron un consentimientos informado. La mayoría de los ensayos fueron estudios enmascarados a doble ciego, con asignación aleatoria de los tratamientos, cruzados y controlados con placebo y/o una sustancia patrón (anfetamina, meta-clorofenilpiperacina o $\mathrm{mCPP}$ ). Las dosis administradas de MDMA variaron desde $40 \mathrm{mg}$ hasta 150 $\mathrm{mg}(1.7 \mathrm{mg} / \mathrm{kg} ; 140 \mathrm{mg} / 70 \mathrm{~kg})$ en una administración única por vía oral, siendo las más estudiadas las comprendidas entre 100-130 mg. A pesar de que la MDMA es un fármaco racémico, con dos estereoisómeros: el SMDMA y el R-MDMA, en todos los estudios se ha administrado la forma racémica.

El objetivo global fue estudiar los efectos farmacológicos agudos de la MDMA, evaluando constantes vitales (presión arterial, frecuencia cardíaca, temperatura, diámetro pupilar), electrocardiograma, efectos subjetivos (incluyendo escalas para medir euforia, sedación, estimulación, cambios en las percepciones, alucinaciones), y rendimiento psicomotor. Además se han determinado cambios en concentraciones de hormonas (ACTH, cortisol, prolactina, dehidroepiandrosterona, vasopresina) (Henry et al, 1998; Forsling et al, 2001) o parámetros inmunológicos (Pacifici et al, 1999; Pacifici et al, 2000; Pacifici et al, 2001a; Pacifici et al, 2001b; Pacifici et al, 2002). En varios se obtuvieron muestras de sangre y orina para analizar las concentraciones de la MDMA y sus metabolitos, y así definir sus parámetros farmacocinéticos)(Fallon et al, 1999). En algunos de los ensayos se incluyó la medición de varias de las variables antes descritas (Grob et al, 1996; Vollenweider et al, 1998; Mas et al, 1999; Camí et al, 2000; de la Torre et al, 2000b; Lester et al, 2000; Tancer y Johanson, 2001; Harris et al, 2002). También se ha estudiado la actividad cerebral mediante electroencefalografía (reflejo de sobresalto y LORETA) o los cambios mediante tomografía de emisión de positrones (Chang et al, 2000; Frei et al, 2000; Gamma et al, 2000; Liechti et al, 2001 b). Dada la complejidad de los diseños y el elevado número de variables estudiadas, cada ensayo clínico ha dado lugar a más de una publicación.

Existen varios estudios en los que se intentó evaluar el posible mecanismo de acción de la MDMA, administrando se forma simultánea otros fármacos que podrían bloquear o aumentar sus efectos (haloperidol, citalopram, ketanserina, paroxetina)(Liechti y Vollenweider, 2001; Farré et al, 2002) y dos estudios de la interacción de la MDMA y el etanol (Hernández-López et al, 2002; Samyn y cols, 2002).

Los resultados de los estudios controlados han permitido confirmar y profundizar en los conocimientos previos, originados en intoxicaciones, encuestas y estudios no controlados, sobre los efectos farmacológicos de la MDMA (Camí y Farré, 1996; Farré et al, 2001a). También han aportado datos origina- 
les y desconocidos hasta la fecha y que han permitido comprender aspectos oscuros de la intoxicación por MDMA. En el terreno de la farmacocinética, se han podido determinar por primera vez los parámetros farmacocinéticos de la MDMA y conocer su metabolismo, y excreción (Kraemer y Maurer, 2002).

En cuanto a la seguridad de los participantes, no se han comunicado efectos indeseables graves ni que obligaran al abandono o retirada de los voluntarios. Tampoco se requirió tratamiento de los posibles efectos indeseables. De hecho las reacciones adversas fueron las descritas en la literatura y similares a las que habían presentado previamente los participante en experiencias al margen de los estudios tras su consumo recreativo. El seguimiento de algunos sujetos mediante PET demostró que la participación en los experimentos no produjo signos de una posible neurotoxicicidad (Vollenweider et al, 2000; Vollenweider y Ludewig, 2002). Tampoco parece que la participación en los estudios incrementara el uso, o indujera el abuso o la dependencia.

A continuación se describirán de forma resumida los principales hallazgos obtenidos en los estudios controlados de administración de MDMA. Para su mejor comprensión se agruparán en las siguientes categorías: fisiológicos, subjetivos, hormonales, inmunológicos y farmacocinéticos.

\section{EFECTOS FISIOLÓGICOS}

Los efectos sobre la presión y frecuencia cardíaca se inician unos 30 minutos tras la dosis y son máximos a los 60 minutos, desapareciendo a las 3-4 horas postadministración (Mas et al, 1999; de la Torre et al, 2000a; Lester et al, 2000). Estos efectos son dosis dependientes. La administración de MDMA produce incrementos de la presión arterial sistólica (PAS) y diastólica (PAD). A dosis de 50, 100, 125 y $150 \mathrm{mg}$ los aumentos de la PAS fueron en promedio de 8, 30, 35 y 40 $\mathrm{mmHg}$. Los incrementos en la PAD fueron menores con un máximo de $20 \mathrm{mmHg}$ para la dosis de $150 \mathrm{mg}$. A dosis de $100 \mathrm{mg} \mathrm{y}$ superiores casi la mitad de los participantes presentaron durante unos minutos criterios de hipertensión arterial (PAS>140 mmHg y y/o PAD >90 mmHg).

En cuanto a la frecuencia cardíaca, a dosis de $50,100,125$ y 150 mg los aumentos fueron en promedio de 10, 20, 24 y 32 latidos por minuto. A dosis de $100 \mathrm{mg}$ y superiores casi la mitad de los participantes presentaron durante unos minutos criterios de taquicardia sinusal (>100 lat/min). La MDMA incrementa en consumo miocárdico de oxígeno sin efectos inotrópicos (Lester et al, 2000). Puede incrementar el intervalo QTc en el electrocardiograma (Farré et al, 2000a).

La temperatura corporal aumenta tras la administración de MDMA, aunque en la mayoría de ensayos estos aumentos no alcanzaron significación estadística frente a placebo. Los aumentos oscilaron entre 0.3 a $0.95{ }^{\circ} \mathrm{C}$ para las dosis bajas y altas, respectivamente (de la Torre et al, 2000a). Posiblemente los estudios no tienen suficiente potencia estadística para demostrar diferencias significativas. En los ensayos clínicos a dosis múltiple se alcanzan incrementos superiores a $1^{\circ} \mathrm{C}$ tras la segunda administración (Farré et al, 2000b; Farré et al, 2001 b). Este incremento de la temperatura es uno de los factores de la hipertermia observada en casos de intoxicación.

La MDMA produce una midriasis intensa, que aparece de forma paralela a las otras manifestaciones fisiológicas pero que permanece hasta unas seis horas tras la administración (Mas et al, 1999).

Los efectos indeseables que más frecuentemente aparecen tras la administración controlada de MDMA son sensación de frío inicial y después calor, temblor, tensión muscular y bruxismo, sequedad de boca, sed, inestabilidad, dificultad para concentrarse y pérdida de apetito. Los efectos indeseables a corto plazo (hasta 24 horas postadministración) más frecuentes son: fatiga, pesadez de piernas, boca seca, pérdida de apetito, insomnio, somnolencia, debilidad, 
tensión muscular, falta de energía, dificultad para concentrarse y cefalea (Vollenweider et al, 1998; Liechti y Vollenweider, 2001). Los que aparecen hasta una semana después del son fatiga, irritabilidad, falta de energía y tensión muscular. Todos estos efectos son similares a los descritos en las encuestas por los consumidores (Camí y Farré, 1996; Farré et al, 2001a).

\section{EFECTOS SUBJETIVOSY PSICOMOTORES}

La MDMA produce sensación de euforia, bienestar, estimulación, energía, extroversión y humor expansivo. Los efectos se inician a los 30-45 minutos, son máximos a las 1-2 horas y desaparecen a las 4 horas después de la administración. Aparecen alteraciones de las percepciones con incrementos en la agudeza auditiva y cambios en el brillo de los objetos. No se han descrito ilusiones ni alucinaciones en los estudios controlados (Vollenweider et al, 1998; Camí et al, 2000; Liechti y Vollenweider, 2001; Tancer y Johanson, 2001; Harris et al, 2002).

A dosis menores de $120 \mathrm{mg}$ la MDMA parece comportarse como un psicoestimulante pero a diferencia de la anfetamina no produce una mejoría del rendimiento psicomotor. A dosis de 125-150 mg aparece, en los momentos de efectos máximos o subidón, sensación de sedación y una disminución leve del rendimiento psicomotor (Camí et al, 2000).

Los efectos empatógenos o entactógenos de la MDMA se han evaluado de forma indirecta debido a la inexistencia de instrumentos de evaluación para esos efectos.

Tras la administración de MDMA se observa en el EEG un aumento de la actividad beta y una disminución de la alfa (Frei et al, 2001).

\section{EFECTOS HORMONALES E INMUNOLÓ- GICOS}

La MDMA incrementa de forma dosis dependiente las concentraciones de ACTH, cortisol, prolactina, dihidroepiandrostrerona y vasopresina, sin aumentar las de hormona del crecimiento (Grob et al, 1996; Mas et al, 1998; Harris et al, 2002). Los incrementos de prolactina están relacionados con la activación serotonérgica, mientras que los de cortisol podrían deberse a incrementos de de noradrenalina y dopamina. Se ha demostrado que la MDMA a dosis bajas produce una elevación significativa de la vasopresina (hormona antidiurética) y una reducción leve de la natremia (Henry et al, 1998; Forsling et al, 2001). Esta podría ser la responsable de los casos de intoxicaciones graves que cursan como un síndrome de secreción inadecuada de hormona antidiurética (SIADH).

La MDMA tiene propiedades inmunomoduladoras, con aumentos significativos en el número de células asesinas naturales (natural killer) y una disminución del número de linfocitos CD4. La MDMA reduce la producción de interleucina-2 e interferón-alfa y aumenta de las concentraciones de interleucinas 4 y 10. Los resultados podrían interpretarse como que la MDMA es un inmunosupresor (Pacifici et al, 1999; Pacifici et al, 2000; Pacifici et al, 2001a; Pacifici et al, 2001b; Pacifici et al, 2002). Quedan por determinar los efectos tras dosis repetidas o administración crónica.

\section{INTERACCIONES FARMACOLÓGICAS}

La MDMA se consume habitualmente en combinación con otras substancias, posiblemente la más frecuente es el etanol. La administración de MDMA junto con etanol provocó mayores efectos cardiovasculares y euforia que la MDMA sola. La MDMA redujo la sensación subjetiva de sedación y de peor rendimiento producida por el alcohol, pero no modificó la alteración del rendimiento psicomotor medido por pruebas objetivas. Los 
resultados parecen indicar que la combinación podría incrementar el riesgo de accidentes al creer los sujetos que están mejor de lo realmente están en cuanto a sedación y rendimiento (Hernández-López et al, 2002).

Los estudios del grupo de Vollenweider han intentado evaluar los mecanismos responsables de los efectos fisiológicos y subjetivos de la MDMA (Liechti y Vollenweider, 2001). La administración previa de haloperidol (1.4 mg i.v.) redujo los efectos subjetivos positivos (euforia) de la MDMA sin modificar el resto de sensaciones subjetivas ni tampoco los efectos cardiovasculares (Liechti y Vollenweider, 2000a). La ketanserina (50 mg oral) redujo los cambios inducidos por la MDMA en las percepciones y la excitación emocional (Liechti et al, 2000b). La administración previa de citalopram (40 mg i.v.), un inhibidor selectivo de la recaptación de serotonina, redujo marcadamente los efectos subjetivos de la MDMA (humor positivo, extraversión, auto-confianza) y disminuyó los efectos cardiovasculares (Liechti et al, 2000a; Liechti y Vollenweider, 2000b). Unos resultados similares se han descrito cuando se administraron simultáneamente paroxetina y MDMA (Farré et al, 2002). El mecanismo de la interacción podría ser una reducción de la unión de la MDMA la transportador de membrana. Como resumen, parece que los efectos euforizantes de la MDMA podrían depender de sus acciones dopaminérgicas mientras que los efectos perceptuales de la actividad serotonérgica.

La MDMA produce efectos más intensos en las mujeres que en los hombres según sugieren los resultados de los diferentes estudios de Vollenweider (Liechti et al, 2001a), aunque no se han realizado estudios planificados de comparación directa.

Tabla 2. Parámetros farmacocinéticos básicos de la MDMA y metabolitos tras la administración de una dosis única oral de 100 mg de MDMA (Segura et al, 2001).

\begin{tabular}{llllll} 
& Cmax $(\mathrm{ng} / \mathrm{ml})$ & $\operatorname{tmax}(\mathrm{h})$ & $\mathrm{ka}{ }^{*}(\mathrm{~h}-1)$ & $\mathrm{ke}(\mathrm{h}-1)$ & $\mathrm{t} 1 / 2(\mathrm{~h})$ \\
\hline MDMA & 222.5 & 2.3 & 2.7 & 0.081 & 9.0 \\
\hline MDA & 13.1 & 6.7 & 0.6 & 0.035 & 24.9 \\
\hline HHMA & 154.5 & 1.2 & 5.3 & 0.063 & 13.4 \\
\hline HMMA & 236.7 & 2.3 & 2.3 & 0.064 & 11.2 \\
\hline HMA & 7.5 & 8.2 & 0.4 & 0.023 & 37.4 \\
\hline
\end{tabular}

* constante de formación metabólica para la MDA, HHMA, HMMA y HMA;

Cmax = concentración máxima plasmática;

tmax = tiempo al que se alcanza la concentración máxima plasmática; ka = constante de absorción;

$\mathrm{ke}=$ constante de eliminación;

t1/2 = semivida de eliminación

Tabla 3. Recuperación urinaria de la MDMA y metabolitos tras la administración de una dosis única de $100 \mathrm{mg}$ de MDMA. El período de recolección fue de 24 horas. Las cantidades se expresan como cantidad total $(\mu \mathrm{mol})$ y en el período acumulado también como porcentaje de la dosis (abreviaturas en el texto) (Segura et al, 2001; Pizarro et al, 2003).

\begin{tabular}{|c|c|c|c|c|c|}
\hline & \multicolumn{5}{|c|}{ Período de recolección } \\
\hline & $0-4 \mathrm{~h}$ & $4-8 \mathrm{~h}$ & $8-12 \mathrm{~h}$ & $12-24 \mathrm{~h}$ & 0-24 h (acumulado) \\
\hline MDMA & 22.7 & 15.5 & 8.8 & 30.8 & $77.8(15.0 \%)$ \\
\hline HMMA & 35.4 & 30.3 & 11.7 & 40.0 & $117.4(22.7 \%)$ \\
\hline HHMA & 30.9 & 21.4 & 12.8 & 26.6 & $91.8(17.7 \%)$ \\
\hline MDA & 1.4 & 1.1 & 0.8 & 4.6 & $7.8(1.5 \%)$ \\
\hline HMA & 1.5 & 1.4 & 0.9 & 3.2 & 7. $(1.35 \%)$ \\
\hline
\end{tabular}




\section{FARMACOCINÉTICA}

Los estudios realizados por el grupo del IMIM de Barcelona constituyen la aportación más relevante para el conocimiento de la farmacocinética y metabolismo de la MDMA tras su administración controlada (Farré y de la Torre, 2002). La MDMA se absorbe rápidamente por vía oral alcanzando las concentraciones máximas a la 1-2 horas postadministración (Mas et al, 1999). Las concentraciones de sus metabolitos 3,4-dihidroximetanfetamina (HHMA) y 4-hidroxi-3-metoximetanfetamina (HMMA) son mayores incluso que las del MDMA y aparecen en sangre antes que la MDMA lo que sugiere un fenómeno de metabolismo de primer paso (Segura et al, 2001). Las principales vías metabólicas de la MDMA pueden verse en la figura 1, siendo la más importante la desmetilenación que formará HHMA, de la que es responsable en un 60\% el citocromo P-450 2D6 (CYP2D6) y después la metilación por la catecolmetiltransferasa (COMT) que producirá HMMA. La transformación mediante desmetilación en MDA es minoritaria (5\%), la MDA será a su vez desmetilenada y metilada de forma similar a la MDMA para formar 3,4-dihidroxianfetamina (HHA) y 4-hidroxi-3-metoxianfetamina (HMA). La relevancia clínica del metabolismo por el CYP2D6 es menor de lo

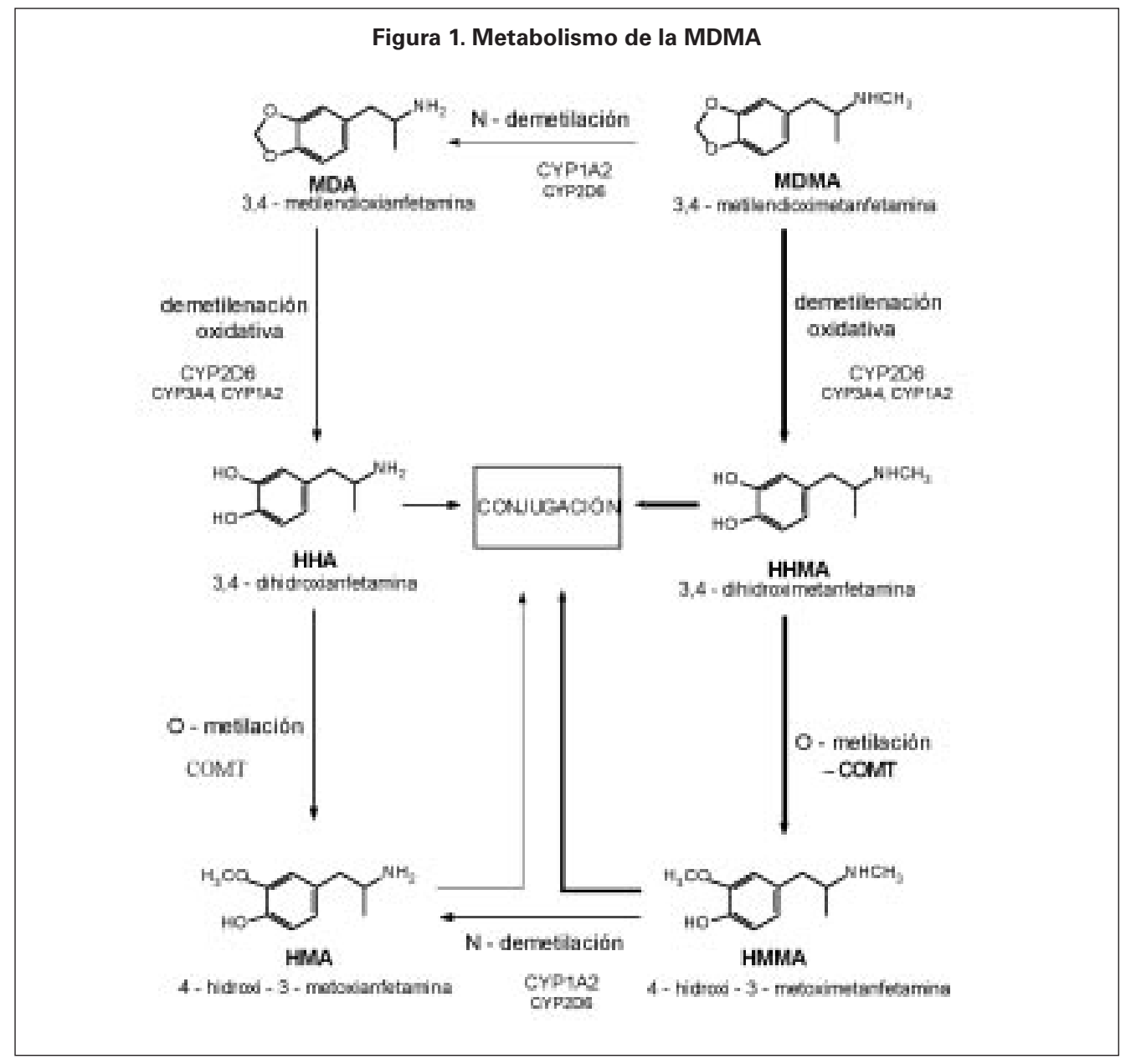


esperado al existir vías metabólicas alternativas por el CYP3A4 y el CYP1A2 (Kraemer y Maurer, 2002). Los metabolitos HMMA, HMA son más activos que la MDMA "in vitro" como liberadores de vasopresina y oxitocina, siendo la HHMA menos activa (Forsling et al, 2002).

Los principales parámetros farmacocinéticos de la MDMA y sus metabolitos tras su administración a dosis única de la MDMA pueden verse en la Tabla 2 (Mas et al, 1999; de la Torre et al, 2000b; Segura et al, 2001; Pizarro et al, 2002b). La recuperación urinaria de MDMA y metabolitos se muestra en la Tabla 3 (Segura et al, 2001; Pizarro et al, 2002b; Segura et al, 2002).

Parece existir una farmacocinética no lineal para la MDMA, es decir que las concentraciones plasmáticas no son proporcionales a la dosis administrada, existiendo una tendencia a la acumulación a dosis altas (de la Torre et al, 1999; de la Torre et al 2000a). Esto se debe a que la MDMA o alguno de sus metabolitos actúan como inhibidores enzimáticos del CYP 2D6. Se inhibiría por ello de forma parcial la biotransformación de MDMA a HHMA y HMMA. Cuando se administraron dos dosis consecutivas de MDMA, separadas entre ellas por intervalos de dos, cuatro y veinticuatro horas, la primera dosis redujo casi a la mitad la formación correspondiente a la segunda dosis de HHMA y HMMA, y por el contrario, incrementó las concentraciones de MDMA entre un 20-40\% (Farré et al, $2001 b)$. Cuando en lugar de una primera dosis de MDMA se administró paroxetina, un conocido inhibidor del CYP2D6, los resultados fueron similares y por ello apoyan la posible acción inhibidora de la MDMA sobre su propio metabolismo y por extrapolación del metabolismo de otros sustratos de este citocromo (Farré et al, 2002).

La farmacocinética de la MDMA es estereoselectiva, así las concentraciones plasmáticas y urinarias del isómero R-MDMA son muy superiores a las del S-MDMA en una proporción de 2:1 aproximadamente. Las concentraciones de S-HMMA y R-HMMA son similares tanto en plasma como en orina
(Fallon et al, 1999; Pizarro et al, 2002a; Pizarro et al, 2002b; Pizarro et al, 2003).

La MDMA alcanza concentraciones elevadas, mayores que las plasmáticas, en la saliva y sudor (Navarro et al, 2001; Pichini et al, 2002; Samyn et al, 2002). Estos fluidos pueden ser de utilidad para el diagnóstico de la intoxicación aguda como alternativa a la sangre u orina.

Como conclusión, los estudios controlados con administración de MDMA en humanos han permitido comprobar algunos de los efectos que describen los consumidores en el contexto festivo y han permitido conocer aspectos novedosos de la farmacología de la MDMA. También han facilitado la comprensión de algunas de las peculiaridades de la intoxicación aguda por esta sustancia. Así se ha confirmado su elevado potencial de abuso debido a la producción de una intensa euforia y sensación de bienestar. En los estudios experimentales no se han podido comprobar los posibles efectos alucinógenos pero sí cambios en las percepciones y la inducción de empatía. Son contribuciones originales de los estudios experimentales el descubrimiento de las acciones hormonales e inmunológicas de la MDMA, que no estaban previamente descritas o que sólo lo estaban de forma parcial. Por ejemplo en el caso de la intoxicación, se han encontrado casos de secreción inadecuada de hormona antidiurética y edema cerebral. En los estudios controlados se ha podido confirmar que la MDMA produce un aumento de la secreción de hormona antidiurética, que podría explicar la toxicidad. En otro campo, estos estudios han permitido comparar las acciones de la MDMA con otras sustancias psicoactivas (anfetamina, mCPP) y relacionar la dosis con la respuesta farmacológica. Los ensayos controlados de interacciones farmacológicas han permitido conocer la influencia del sistema dopaminérgico y serotonérgico en los efectos de la MDMA. Tal vez la contribución más relevante de los estudios controlados es la aportación a la farmacocinética de la MDMA. Se han podido determinar sus principales parámetros farmacocinéticos, sus principales metabolitos y su 
perfil de excreción urinaria. Se ha realizado estudios de distribución de la MDMA en saliva y sudor, que han sido útiles para estandarizar los sistemas de detección rápida de anfetaminas en estos fluidos. Además se ha demostrado su farmacocinética no-lineal y su capacidad de inhibir al citocromo P-4502D6. Estas dos características implican un incremento desproporcionado de las concentraciones de MDMA tras dosis altas o repetidas y la posibilidad de interacciones con otros sustratos de este sistema metabólico, que resulte en un incremento de la toxicidad.

En el caso de la MDMA y demás anfetaminas de diseño, quedan aún muchas lagunas por estudiar y muchos estudios que realizar, cuyos diseños, tanto experimentales o controlados como observacionales deberán ayudarnos en un futuro a determinar científicamente algunos problemas que aún no están aclarados en la toxicidad a corto, medio y largo plazo de esta sustancia.

Agradecimientos: Este proyecto ha sido financiado en parte por el Fondo de Investigación Sanitaria (FIS 97/1198; Fis 98/018; FIS 01/1336) y la Generalitat de Catalunya (Soporte a los grupos de investigación consolidados en Catalunya, CIRIT 1999SGR 00242, 2001SGR00407).

\section{REFERENCIAS}

Baños JE, Farré M. Principios de Farmacología Clínica. Bases científicas de la utilización de medicamentos. Barcelona: Masson, 2002.

Camí J, Farré M. Extasis, la droga de la ruta del bakalao. Med Clín (Barc) 1996; 106:711-76.

Camí J, Farré M, Mas M, Roset PN, Poudevida S, Mas A, San L, de la Torre R,. Human pharmacology of 3,4-Methylenedioxymethamphetamine ("Ecstasy"): Psychomotor performance and subjective effects. J Clin Psychopharmacol 2000; 20:455-466.

Chang L, Grob CS, Ernst T, Itti L, Mishkin FS, JoseMelchor R, Poland RE. Effect of ecstasy [3,4methylenedioxymethamphetamine (MDMA)] on cerebral blood flow: a co-registered SPECT and MRI study. Psychiat Res Neuroimaging 2000: 98:15-28

De la Torre R, Farré M, Ortuño J, Mas M, Brenneisen R, Roset PN, Segura J, Camí J. Non-linear pharmacokinetics of MDMA ('ecstasy') in humans. Br J Clin Pharmacol, 2000a;49:104-9.

De la Torre R, Farré M, Roset PN, Hernández-López C, Mas M, Ortuño J, Menoyo E, Pizarro N, Segura J, Cami J. Pharmacology of MDMA in humans. An NY Acad Sci 2000b;914:225-37.

De la Torre R, Ortuño J, Mas M, Farre M, Segura J. Fatal MDMA intoxication. Lancet. 1999;13;353:593

Fallon JK, Kicman AT, Henry JA, Milligan PJ, Cowan DA, Hut AJ. Stereospecific analysis and enantiomeric disposition of 3, 4-methylenedioxymethamphetamine (Ecstasy) in humans. Clin Chem 1999;45:1058-69.

Farré M, de la Torre R. Pharmacokinetics of MDMA in humans: toxicological relevance. Adiktologie 2002;1 (suppl): 32-34.

Farré M, Mas M, Molina L, Camí J. Long QT syndromes induced by ecstasy and other recreational drugs. Electronic letters to Heart. Heart 2000a; 83:627-633. (http://heart.bmjjournals.com/cgi/eletters/83/627, consultada 30-092000)

Farré M, Roset PN, de la Torre R, Poudevida S, Hernández-López C, Peiró AM, Menoyo E, Sánchez I, Camí J. Farmacología clínica de la 3,4metilenodioximetanfetamina (MDMA, éxtasis). Conductas Adictivas 2001a; 1: 1-4 (www.conductas adictivas.org; consultado 18-03-2002).

Farré M, Roset PN, Hernández-López C, Poudevida S, Menoyo E, de la Torre R, Ortuño J, Peiró A, Camí J. Repeated administration of MDMA to healthy volunteers. Drug Alcohol Depend 2001 b;63 (suppl):175.

Farré M, Roset PN, Tomillero A, Hernández-López C, Poudevida S, de la Torre R, Ortuño J, Camí J. MDMA repeated administration in humans: a dose-finding pilot study. Drug Alcohol Depend 2000b;60 (suppl 1):173.

Farré M, Roset P.N, de la Torre R, Segura M, Ortuño J, Peiró AM, Torrens M, Pacifici R, Zuccaro P, Camí J. Interaction of paroxetine and MDMA in humans. Drug Alcohol Depend 2002; 66 (suppl 1):196.

Forsling M, Fallon JK, Kicman AT, Hutt AJ, Cowan DA, Henry JA.. Arginine vasopressin release in 
response to the administration of 3,4-methylenedioxymethamphetamine ("ecstasy"): is metabolism a contributory factor?. J Pharm Pharmacol 2001;53:1357-63.

Forsling ML, Fallon JK, Shah D, Tilbrook GS, Cowan DA, Kicman AT, Hutt AJ. The effect of 3,4methylenedioxymethamphetamine (MDMA, 'ecstasy') and its metabolites on neurohypophysial hormone release from the isolated rat hypothalamus. $\mathrm{Br} J$ Pharmacol 2002;135:649-56.

Frei E, Gamma A, Pascual-Marqui R, Lehmann D, Hell D, Vollenweider FX.

Localization of MDMA-induced brain activity in healthy volunteers using low resolution brain electromagnetic tomography (LORETA). Hum Brain Mapp 2001; 14:152-65.

Gamma A, Buck A, Berthold T, Liechti ME, Vollenweider FX. 3,4-Methylenedioxymethamphetamine (MDMA) modulates cortical and limbic brain activity as measured by [H(2)(15)O]-PET in healthy humans. Neuropsychopharmacology 2000;23:388-95.

Gouzoulis-Mayfrank E, Schreckenberger M, Sabri O, Arning C, Thelen B, Spitzer M, Kovar KA, Hermle L, Bull U, Sass H. Neurometabolic effects of psilocybin, 3,4- methylenedioxyethylamphetamine (MDE) and dmethamphetamine in healthy volunteers. A double-blind, placebocontrolled PET study with [18F]FDG. Neuropsychopharmacology 1999a;20:565-81.

Gouzoulis-Mayfrank E, Thelen B, Habermeyer E, Kunert HJ, Kovar KA, Lindenblatt H, Hermle L, Spitzer M, Sass H. Psychopathological, neuroendocrine and autonomic effects of 3,4methylenedioxyethylamphetamine (MDE), psilocybin and d-methamphetamine in healthy volunteers. Results of an experimental doubleblind placebo- controlled study. Psychopharmacology (Berl) 1999b;142:41-50.

Grob CS, Poland RE, Chang L, Ernst T. Psychobiologic effects of 3,4-methylenedioxymethamphetamine in humans: methodological considerations and preliminary observations. Behav Brain Res 1996;73:103-7.

Harris DS, Baggott M, Mendelson JH, Mendelson JE, Jones RT. Subjective and hormonal effects of 3,4-methylenedioxymethamphetamine (MDMA) in humans. Psychopharmacology (Berl) 2002;162:396-405.

Henry JA, Fallon JK, Kicman AT, Hutt AJ, Cowan DA, Forsling M. Low-dose MDMA ("ecstasy") induces vasopressin secretion. Lancet 1998:351:1784.

Hernández-López C, Farré M, Roset PN, Menoyo E, Pizarro N, Ortuño J, Torrens M, Camí J, de La Torre R. 3,4-Methylenedioxymethamphetamine (ecstasy) and alcohol interactions in humans: psychomotor performance, subjective effects, and pharmacokinetics. J Pharmacol Exp Ther 2002;300:236-44.

Holland J. The history of MDMA. En: Holland J, editor. Ecstasy: the complete guide. Rochester: Park Street Press; 2001. p. 11-20.

Kleinman AM, Holland J. Clinical research with MDMA: a worldwide review. En: Holland J, editor. Ecstasy: the complete guide. Rochester: Park Street Press; 2001. p. 297-316.

Kraemer T, Maurer HH (2002) Toxicokinetics of amphetamines: metabolism and toxicokinetic data of designer drugs, amphetamine, methamphetamine, and their $\mathrm{N}$-alkyl derivatives. Ther Drug Monit 24: 277-89.

Lester SJ, Baggott M, Welm S, Schiller NB, Jones RT, Foster E, Mendelson J.

Cardiovascular Effects of 3,4-Methylenedioxymethamphetamine. A Double-

Blind, Placebo-Controlled Trial. Ann Intern Med 2000;133:969-73

Liechti ME, Baumann C, Gamma A, Vollenweider FX. Acute psychological effects of 3,4-methylenedioxymethamphetamine (MDMA, "Ecstasy") are attenuated by the serotonin uptake inhibitor citalopram. Neuropsychopharmacology 2000a; 22:513-21.

Liechti ME, Gamma A, Vollenweider FX. Gender differences in the subjective effects of MDMA. Psychopharmacology (Berl) 2001a;154:161-8.

Liechti ME, Geyer MA, Hell D, Vollenweider FX. Effects of MDMA (ecstasy) on prepulse inhibition and habituation of startle in humans after pretreatment with citalopram, haloperidol, or ketanserin. Neuropsychopharmacology 2001 b; 24(3):240-52.

Liechti ME, Saur MR, Gamma A, Hell D, Vollenweider FX. Psychological and physiological effects of MDMA ("Ecstasy") after pretreatment with the 5-HT(2) antagonist ketanserin in healthy humans. Neuropsychopharmacology 2000b; 23:396-404.

Liechti ME, Vollenweider FX. Acute psychological and physiological effects of MDMA ("Ecstasy") after haloperidol pretreatment in healthy 
humans. Eur Neuropsychopharmacol 2000a;10:289-95.

Liechti ME, Vollenweider FX. The serotonin uptake inhibitor citalopram reduces acute cardiovascular and vegetative effects of 3,4-methylenedioxymethamphetamine ('Ecstasy') in healthy volunteers. J Psychopharmacol 2000b;14:26974.

Liechti ME, Vollenweider FX. Wich receptor mediate subjective effects of MDMA in humans?. A summary of mechanistic studies. Hum Psychopharmacol Clin Exp 2001;16:589-98.

Mas M, Farré M, de la Torre R, Roset PN, Ortuño J, Segura J, Camí J.

Cardiovascular and neuroendocrine effects and pharmacokinetics of 3, 4-methylenedioxymethamphetamine in humans. J Pharmacol Exp Ther 1999;290: 136-45.

Navarro M, Pichini S, Farre M, Ortuño J, Roset PN, Segura J, de la Torre R. Usefulness of saliva for measurement of 3,4- methylenedioxymethamphetamine and its metabolites: correlation with plasma drug concentrations and effect of salivary pH. Clin Chem 2001;47:1788-95.

Pacifici R, Zuccaro P, Farré M, Pichini S, Di Carlo S, Roset PN, Hernández López C, Ortuno J, Segura J, Cami J, de la Torre R. Immunomodulating activity of MDMA Ann N Y Acad Sci 2000;914:215-24.

Pacifici R, Zuccaro P, Farré M, Pichini S, Di Carlo S, Roset PN, Ortuño J, Pujadas M, Bacosi A, Menoyo E, Segura J, de la Torre R. Effects of repeated doses of MDMA ("ecstasy") on cellmediated immune response in humans. Life Sci 2001a;69:2931-41.

Pacifici R, Zuccaro P, Farré M, Pichini S, Di Carlo S, Roset PN, Ortuño J, Segura J, de la Torre R. Immunomodulating properties of MDMA alone and in combination with alcohol: a pilot study. Life Sci 1999;65:309-16.

Pacifici R, Zuccaro P, Farré M, Pichini S, Di Carlo S, Roset PN, Palmi I, Ortuño J,

Menoyo E, Segura J, de la Torre R. Cell-mediated immune response in MDMA users after repeated dose administration: studies in controlled versus noncontrolled settings. Ann NY Acad Sci 2002;965:421-33.

Pacifici R, Zuccaro P, Hernández-López, Pichini S, di Carlo S, Farré M, Roset PN, Ortuño J, Segura J, de la Torre R. Acute effects of MDMA alone and in combination with ethanol on the immu- ne system in humans. J Pharmacol Exp Ther 2001b;296:207-15.

Pichini S, Navarro M, Farré M, Ortuño J, Roset PN, Pacifici R, Zuccaro P, Segura J, de la Torre R. On-site testing of 3,4-methylenedioxymethamphetamine (ecstasy) in saliva with Drugwipe and Drugread: a controlled study in recreational users. Clin Chem 2002;48:174-6.

Pizarro N, de la Torre R, Farré M, Segura J, Llebaría A, Joglar J Synthesis and Capillary Electrophoresis Analysis of enantiomerically enriched reference standards of MDMA and its main metabolites Bioorganics and Medicinal Chemistry 2002a;10:1085-1092.

Pizarro N, Llebaría A, Cano S, Joglar J, Farré M, Segura J, de la Torre R. Stereochemical analysis of 3,4-methylenedioxymethamphetamine and its main metabolites by gas chromatography/mass spectrometry. Rapid Commun Mass Spectrom. 2003;17:330-6.

Pizarro N, Ortuño J, Farré M, Hernández-López C, Pujadas M, Llebaría A, Joglar J, Roset PN, Mas M, Segura J, Cami J, de la Torre R. Determination of MDMA and its metabolites in blood and urine by gas chromatography-mass spectrometry and analysis of enantiomers by capillary electrophoresis. J Anal Toxicol 2002b;26:15765.

Samyn N, De Boeck G, Wood M, Lamers CTJ, De Waard D, Brookhuis KA, Verstraete AG, Riedel WJ. Plasma, oral fluid and sweat ecstasy concentrations in controlled and real life conditions. Forensic Sci Int 2002;128:90-97.

Segura M, Ortuño J, Farré M, McLure JA, Pujadas M, Pizarro N, Llebaría A, Joglar J, Roset PN, Segura J, de La Torre R (2001) 3,4-Dihydroxymethamphetamine (HHMA). A Major in Vivo 3,4- methylenedioxymethamphetamine (MDMA) Metabolite in Humans. Chem Res Toxicol 2001;14:1203-8.

Segura M, , Ortuño J, McLure JA, Pujades MA, Pizarro N, Farré M, Llebaria A, Joglar J, Segura J, de la Torre R. High-performance liquid chromatography with electrochemical detection applied to the analysis of 3,4-dihydroxymethamphetamine in human plasma and urine. J Chromatography B 2002;769:313-21.

Tancer ME, Johanson CE (2001) The subjective effects of MDMA and MCPP in moderate MDMA users. Drug Alcohol Depend 65: 97-101

Vollenweider FX, Gamma A, Liechti M, Huber T. Psychological and cardiovascular effects and 
short-term sequelae of MDMA ("ecstasy") in MDMA naive healthy volunteers. Neuropsychopharmacology 1998;19:241-51.

Vollenweider FX, Gucker P, Schönbächler R, Kamber E, Vollenweider-Scherpenhuyzen MFI, Schubiger G, Hell D. Effects of MDMA on 5-

HT uptake sites using PET and [11C]-McN5652 in humans. 2000. Data presented at 2000 confe- rence of the German Society for Psychiatry, Psychotherapy and Neuromedicine [Deutsche Gesellschaft für psychiatrie, Psychotherapie und Nervenheilkunde].

Vollenweider FX, Ludewig S. Neurocognitive effects of MDMA: a summary of controlled human studies. Adiktologie 2002; 1 (suppl):4752. 